\title{
INEQUALITIES OF HERMITE-HADAMARD TYPE FOR COMPOSITE CONVEX FUNCTIONS
}

\author{
SILVESTRU SEVER DRAGOMIR ${ }^{1,2}$
}

\begin{abstract}
In this paper we obtain some inequalities of Hermite-Hadamard type for composite convex functions. Applications for $A G, A H$-convex functions, $G A, G G, G H$-convex functions and $H A, H G, H H$-convex function are given. Applications for $p, r$-convex and $\operatorname{LogExp}$ convex functions are presented as well.
\end{abstract}

\section{Introduction}

The following inequality holds for any convex function $f$ defined on $\mathbb{R}$

$$
f\left(\frac{a+b}{2}\right) \leq \frac{1}{b-a} \int_{a}^{b} f(x) d x \leq \frac{f(a)+f(b)}{2}, \quad a, b \in \mathbb{R}, a<b .
$$

It was firstly discovered by Ch. Hermite in 1881 in the journal Mathesis (see [18]). But this result was nowhere mentioned in the mathematical literature and was not widely known as Hermite's result.

E. F. Beckenbach, a leading expert on the history and the theory of convex functions, wrote that this inequality was proven by J. Hadamard in 1893 [3]. In 1974, D. S. Mitrinović found Hermite's note in Mathesis [18]. Since (1.1) was known as Hadamard's inequality, the inequality is now commonly referred as the Hermite-Hadamard inequality.

In order to extend this result for other classes of functions, we need the following preparations.

Let $g:[a, b] \rightarrow[g(a), g(b)]$ be a continuous strictly increasing function that is differentiable on $(a, b)$.

Definition 1. A function $f:[a, b] \rightarrow \mathbb{R}$ will be called composite- $g^{-1}$ convex (concave) on $[a, b]$ if the composite function $f \circ g^{-1}:[g(a), g(b)] \rightarrow \mathbb{R}$ is convex (concave) in the usual sense on $[g(a), g(b)]$.

In this way, any concept of convexity (log-convexity, harmonic convexity, trigonometric convexity, hyperbolic convexity, $h$-convexity, quasi-convexity, $s$-convexity, $s$-Godunova-Levin convexity etc...) can be extended to the corresponding composite- $g^{-1}$ convexity. The details however will not be presented here.

If $f:[a, b] \rightarrow \mathbb{R}$ is composite $-g^{-1}$ convex on $[a, b]$ then we have the inequality

$$
f \circ g^{-1}((1-\lambda) u+\lambda v) \leq(1-\lambda) f \circ g^{-1}(u)+\lambda f \circ g^{-1}(v)
$$

for any $u, v \in[g(a), g(b)]$ and $\lambda \in[0,1]$.

1991 Mathematics Subject Classification. 26D15; 26D10.

Key words and phrases. Convex functions, $A G, A H$-convex functions, $G A, G G, G H$-convex functions and $H A, H G, H H$-convex function, Integral inequalities. 
This is equivalent to the condition

$$
f \circ g^{-1}((1-\lambda) g(t)+\lambda g(s)) \leq(1-\lambda) f(t)+\lambda f(s)
$$

for any $t, s \in[a, b]$ and $\lambda \in[0,1]$.

If we take $g(t)=\ln t, t \in[a, b] \subset(0, \infty)$, then the condition (1.3) becomes

$$
f\left(t^{1-\lambda} s^{\lambda}\right) \leq(1-\lambda) f(t)+\lambda f(s)
$$

for any $t, s \in[a, b]$ and $\lambda \in[0,1]$, which is the concept of $G A$-convexity as considered in $[1]$.

If we take $g(t)=-\frac{1}{t}, t \in[a, b] \subset(0, \infty)$, then (1.3) becomes

$$
f\left(\frac{t s}{(1-\lambda) s+\lambda t}\right) \leq(1-\lambda) f(t)+\lambda f(s)
$$

for any $t, s \in[a, b]$ and $\lambda \in[0,1]$, which is the concept of $H A$-convexity as considered in [1].

If $p>0$ and we consider $g(t)=t^{p}, t \in[a, b] \subset(0, \infty)$, then the condition (1.3) becomes

$$
f\left[\left((1-\lambda) t^{p}+\lambda s^{p}\right)^{1 / p}\right] \leq(1-\lambda) f(t)+\lambda f(s)
$$

for any $t, s \in[a, b]$ and $\lambda \in[0,1]$, which is the concept of $p$-convexity as considered in $[22]$.

If we take $g(t)=\exp t, t \in[a, b]$, then the condition (1.3) becomes

$$
f[\ln ((1-\lambda) \exp (t)+\exp g(s))] \leq(1-\lambda) f(t)+\lambda f(s)
$$

which is the concept of LogExp convex function on $[a, b]$ as considered in [7].

Further, assume that $f:[a, b] \rightarrow J, J$ an interval of real numbers and $k: J \rightarrow \mathbb{R}$ a continuous function on $J$ that is strictly increasing (decreasing) on $J$.

Definition 2. We say that the function $f:[a, b] \rightarrow J$ is $k$-composite convex (concave) on $[a, b]$, if $k \circ f$ is convex (concave) on $[a, b]$.

In this way, any concept of convexity as mentioned above can be extended to the corresponding $k$-composite convexity. The details however will not be presented here.

With $g:[a, b] \rightarrow[g(a), g(b)]$ a continuous strictly increasing function that is differentiable on $(a, b), f:[a, b] \rightarrow J, J$ an interval of real numbers and $k: J \rightarrow \mathbb{R}$ a continuous function on $J$ that is strictly increasing (decreasing) on $J$, we can also consider the following concept:

Definition 3. We say that the function $f:[a, b] \rightarrow J$ is $k$-composite- $g^{-1}$ convex (concave) on $[a, b]$, if $k \circ f \circ g^{-1}$ is convex (concave) on $[g(a), g(b)]$.

This definition is equivalent to the condition

$$
k \circ f \circ g^{-1}((1-\lambda) g(t)+\lambda g(s)) \leq(1-\lambda)(k \circ f)(t)+\lambda(k \circ f)(s)
$$

for any $t, s \in[a, b]$ and $\lambda \in[0,1]$.

If $k: J \rightarrow \mathbb{R}$ is strictly increasing (decreasing) on $J$, then the condition (1.8) is equivalent to:

$$
f \circ g^{-1}((1-\lambda) g(t)+\lambda g(s)) \leq(\geq) k^{-1}[(1-\lambda)(k \circ f)(t)+\lambda(k \circ f)(s)]
$$

for any $t, s \in[a, b]$ and $\lambda \in[0,1]$. 
If $k(t)=\ln t, t>0$ and $f:[a, b] \rightarrow(0, \infty)$, then the fact that $f$ is $k$-composite convex on $[a, b]$ is equivalent to the fact that $f$ is log-convex or multiplicatively convex or $A G$-convex, namely, for all $x, y \in I$ and $t \in[0,1]$ one has the inequality:

$$
f(t x+(1-t) y) \leq[f(x)]^{t}[f(y)]^{1-t} .
$$

A function $f: I \rightarrow \mathbb{R} \backslash\{0\}$ is called $A H$-convex (concave) on the interval $I$ if the following inequality holds [1]

$$
f((1-\lambda) x+\lambda y) \leq(\geq) \frac{1}{(1-\lambda) \frac{1}{f(x)}+\lambda \frac{1}{f(y)}}=\frac{f(x) f(y)}{(1-\lambda) f(y)+\lambda f(x)}
$$

for any $x, y \in I$ and $\lambda \in[0,1]$.

An important case that provides many examples is that one in which the function is assumed to be positive for any $x \in I$. In that situation the inequality (1.11) is equivalent to

$$
(1-\lambda) \frac{1}{f(x)}+\lambda \frac{1}{f(y)} \leq(\geq) \frac{1}{f((1-\lambda) x+\lambda y)}
$$

for any $x, y \in I$ and $\lambda \in[0,1]$.

Taking into account this fact, we can conclude that the function $f: I \rightarrow(0, \infty)$ is $A H$-convex (concave) on $I$ if and only if $f$ is $k$-composite concave (convex) on $I$ with $k:(0, \infty) \rightarrow(0, \infty), k(t)=\frac{1}{t}$.

Following [1], we can introduce the concept of $G H$-convex (concave) function $f: I \subset(0, \infty) \rightarrow \mathbb{R}$ on an interval of positive numbers $I$ as satisfying the condition

$$
f\left(x^{1-\lambda} y^{\lambda}\right) \leq(\geq) \frac{1}{(1-\lambda) \frac{1}{f(x)}+\lambda \frac{1}{f(y)}}=\frac{f(x) f(y)}{(1-\lambda) f(y)+\lambda f(x)} .
$$

Since

$$
f\left(x^{1-\lambda} y^{\lambda}\right)=f \circ \exp [(1-\lambda) \ln x+\lambda \ln y]
$$

and

$$
\frac{f(x) f(y)}{(1-\lambda) f(y)+\lambda f(x)}=\frac{f \circ \exp (\ln x) f \circ \exp (\ln y)}{(1-\lambda) f \circ \exp (y)+\lambda f \circ \exp (x)}
$$

then $f: I \subset(0, \infty) \rightarrow \mathbb{R}$ is $G H$-convex (concave) on $I$ if and only if $f \circ \exp$ is $A H$ convex (concave) on $\ln I:=\{x \mid x=\ln t, t \in I\}$. This is equivalent to the fact that $f$ is $k$-composite- $g^{-1}$ concave (convex) on $I$ with $k:(0, \infty) \rightarrow(0, \infty), k(t)=\frac{1}{t}$ and $g(t)=\ln t, t \in I$.

Following [1], we say that the function $f: I \subset \mathbb{R} \backslash\{0\} \rightarrow(0, \infty)$ is $H H$-convex if

$$
f\left(\frac{x y}{t x+(1-t) y}\right) \leq \frac{f(x) f(y)}{(1-t) f(y)+t f(x)}
$$

for all $x, y \in I$ and $t \in[0,1]$. If the inequality in (1.13) is reversed, then $f$ is said to be $H H$-concave.

We observe that the inequality (1.13) is equivalent to

$$
(1-t) \frac{1}{f(x)}+t \frac{1}{f(y)} \leq \frac{1}{f\left(\frac{x y}{t x+(1-t) y}\right)}
$$

for all $x, y \in I$ and $t \in[0,1]$.

This is equivalent to the fact that $f$ is $k$-composite- $g^{-1}$ concave on $[a, b]$ with $k:(0, \infty) \rightarrow(0, \infty), k(t)=\frac{1}{t}$ and $g(t)=-\frac{1}{t}, t \in[a, b]$. 
The function $f: I \subset(0, \infty) \rightarrow(0, \infty)$ is called $G G$-convex on the interval $I$ of real umbers $\mathbb{R}$ if $[1]$

$$
f\left(x^{1-\lambda} y^{\lambda}\right) \leq[f(x)]^{1-\lambda}[f(y)]^{\lambda}
$$

for any $x, y \in I$ and $\lambda \in[0,1]$. If the inequality is reversed in (1.15) then the function is called $G G$-concave.

This concept was introduced in 1928 by P. Montel [19], however, the roots of the research in this area can be traced long before him [20]. It is easy to see that [20], the function $f:[a, b] \subset(0, \infty) \rightarrow(0, \infty)$ is $G G$-convex if and only if the the function $g:[\ln a, \ln b] \rightarrow \mathbb{R}, g=\ln \circ f \circ \exp$ is convex on $[\ln a, \ln b]$. This is equivalent to the fact that $f$ is $k$-composite- $g^{-1}$ convex on $[a, b]$ with $k:(0, \infty) \rightarrow \mathbb{R}, k(t)=\ln t$ and $g(t)=\ln t, t \in[a, b]$.

Following [1] we say that the function $f: I \subset \mathbb{R} \backslash\{0\} \rightarrow(0, \infty)$ is $H G$-convex if

$$
f\left(\frac{x y}{t x+(1-t) y}\right) \leq[f(x)]^{1-t}[f(y)]^{t}
$$

for all $x, y \in I$ and $t \in[0,1]$. If the inequality in (1.3) is reversed, then $f$ is said to be $H G$-concave.

Let $f:[a, b] \subset(0, \infty) \rightarrow(0, \infty)$ and define the associated functions $G_{f}:\left[\frac{1}{b}, \frac{1}{a}\right] \rightarrow$ $\mathbb{R}$ defined by $G_{f}(t)=\ln f\left(\frac{1}{t}\right)$. Then $f$ is $H G$-convex on $[a, b]$ iff $G_{f}$ is convex on $\left[\frac{1}{b}, \frac{1}{a}\right]$. This is equivalent to the fact that $f$ is $k$-composite- $g^{-1}$ convex on $[a, b]$ with $k:(0, \infty) \rightarrow \mathbb{R}, k(t)=\ln t$ and $g(t)=-\frac{1}{t}, t \in[a, b]$. if

Following [21], we say that the function $f:[a, b] \rightarrow(0, \infty)$ is $r$-convex, for $r \neq 0$,

$$
f((1-\lambda) x+\lambda y) \leq\left[(1-\lambda) f^{r}(y)+\lambda f^{r}(x)\right]^{1 / r}
$$

for any $x, y \in[a, b]$ and $\lambda \in[0,1]$.

If $r>0$, then the condition (1.17) is equivalent to

$$
f^{r}((1-\lambda) x+\lambda y) \leq(1-\lambda) f^{r}(y)+\lambda f^{r}(x)
$$

namely $f$ is $k$-composite convex on $[a, b]$ where $k(t)=t^{r}, t \geq 0$.

If $r<0$, then the condition (1.17) is equivalent to

$$
f^{r}((1-\lambda) x+\lambda y) \geq(1-\lambda) f^{r}(y)+\lambda f^{r}(x)
$$

namely $f$ is $k$-composite concave on $[a, b]$ where $k(t)=t^{r}, t>0$.

In this paper we obtain some inequalities of Hermite-Hadamard type for composite convex functions. Applications for various classes of convexity as provided above are given as well.

\section{Some Refinements}

We need the following refinement of Hermite-Hadamard inequality. This result was obtained for the first time by Barnett, Cerone \& Dragomir in 2002 in the paper [2, p. 10, Eq. (2.2)] where various applications for the Hermite-Hadamard divergence measure in Information Theory were also given. The same result was also rediscovered by El Farissi in 2010 with a similar proof, see [16]. 
Lemma 1. Assume that $h:[c, d] \rightarrow \mathbb{R}$ is convex on $[c, d]$. Then for any $\lambda \in[0,1]$ we have

$$
\begin{aligned}
h\left(\frac{c+d}{2}\right) & \leq \lambda h\left(\frac{\lambda d+(2-\lambda) c}{2}\right)+(1-\lambda) h\left(\frac{(1+\lambda) d+(1-\lambda) c}{2}\right) \\
& \leq \frac{1}{d-c} \int_{c}^{d} h(u) d u \\
& \leq \frac{1}{2}[h((1-\lambda) c+\lambda d)+\lambda h(c)+(1-\lambda) h(d)] \leq \frac{h(c)+h(d)}{2}
\end{aligned}
$$

Proof. For the sake of completeness, we give here a simple proof as in [2]. Applying the Hermite-Hadamard inequality on each subinterval $[c,(1-\lambda) c+\lambda d]$, $[(1-\lambda) c+\lambda d, d]$, where $\lambda \in(0,1)$, then we have,

$$
\begin{aligned}
& h\left(\frac{c+(1-\lambda) c+\lambda d}{2}\right) \times[(1-\lambda) c+\lambda d-c] \\
& \leq \int_{c}^{(1-\lambda) c+\lambda d} h(u) d u \\
& \leq \frac{h((1-\lambda) c+\lambda d)+h(c)}{2} \times[(1-\lambda) c+\lambda d-c]
\end{aligned}
$$

and

$$
\begin{aligned}
& h\left(\frac{(1-\lambda) c+\lambda d+d}{2}\right) \times[d-(1-\lambda) c-\lambda d] \\
& \leq \int_{(1-\lambda) c+\lambda d}^{d} h(u) d u \\
& \leq \frac{h(d)+h((1-\lambda) c+\lambda d)}{2} \times[d-(1-\lambda) c-\lambda d],
\end{aligned}
$$

which are clearly equivalent to

$$
\begin{aligned}
\lambda h\left(\frac{\lambda d+(2-\lambda) c}{2}\right) & \leq \frac{1}{d-c} \int_{c}^{(1-\lambda) c+\lambda d} h(u) d u \\
& \leq \frac{\lambda h((1-\lambda) c+\lambda d)+\lambda h(c)}{2}
\end{aligned}
$$

and

$$
\begin{aligned}
(1-\lambda) h\left(\frac{(1+\lambda) d+(1-\lambda) c}{2}\right) & \leq \frac{1}{d-c} \int_{(1-\lambda) c+\lambda d}^{d} h(u) d u \\
& \leq \frac{(1-\lambda) h(d)+(1-\lambda) h((1-\lambda) c+\lambda d)}{2}
\end{aligned}
$$

respectively.

Summing (2.2) and (2.3), we obtain the second and first inequality in (2.1). 
By the convexity property, we obtain

$$
\begin{aligned}
& \lambda h\left(\frac{\lambda d+(2-\lambda) c}{2}\right)+(1-\lambda) h\left(\frac{(1+\lambda) d+(1-\lambda) c}{2}\right) \\
& \geq h\left[\lambda\left(\frac{\lambda d+(2-\lambda) c}{2}\right)+(1-\lambda)\left(\frac{(1+\lambda) d+(1-\lambda) c}{2}\right)\right] \\
& =h\left(\frac{c+d}{2}\right)
\end{aligned}
$$

and the first inequality in (2.1) is proved.

For various inequalities of Hermite-Hadamard type see the monograph online [8] and the more recent survey paper [6].

If $g$ is a function which maps an interval $I$ of the real line to the real numbers, and is both continuous and injective then we can define the $g$-mean of two numbers $a, b \in I$ as

$$
M_{g}(a, b):=g^{-1}\left(\frac{g(a)+g(b)}{2}\right)
$$

If $I=\mathbb{R}$ and $g(t)=t$ is the identity function, then $M_{g}(a, b)=A(a, b):=\frac{a+b}{2}$, the arithmetic mean. If $I=(0, \infty)$ and $g(t)=\ln t$, then $M_{g}(a, b)=G(a, b):=\sqrt{a b}$, the geometric mean. If $I=(0, \infty)$ and $g(t)=\frac{1}{t}$, then $M_{g}(a, b)=H(a, b):=$ $\frac{2 a b}{a+b}$, the harmonic mean. If $I=(0, \infty)$ and $g(t)=t^{p}, p \neq 0$, then $M_{g}(a, b)=$ $M_{p}(a, b):=\left(\frac{a^{p}+b^{p}}{2}\right)^{1 / p}$, the power mean with exponent $p$. Finally, if $I=\mathbb{R}$ and $g(t)=\exp t$, then

$$
M_{g}(a, b)=L M E(a, b):=\ln \left(\frac{\exp a+\exp b}{2}\right),
$$

the LogMeanExp function.

Theorem 1. Let $g:[a, b] \rightarrow[g(a), g(b)]$ be a continuous strictly increasing function that is differentiable on $(a, b)$. If $f:[a, b] \rightarrow \mathbb{R}$ is composite- $g^{-1}$ convex on $[a, b]$, then

$$
\begin{aligned}
f\left(M_{g}(a, b)\right) & \leq \lambda f \circ g^{-1}\left(\frac{\lambda g(b)+(2-\lambda) g(a)}{2}\right) \\
& +(1-\lambda) f \circ g^{-1}\left(\frac{(1+\lambda) g(b)+(1-\lambda) g(a)}{2}\right) \\
& \leq \frac{1}{g(b)-g(a)} \int_{a}^{b} f(t) g^{\prime}(t) d t \\
& \leq \frac{1}{2}\left[f \circ g^{-1}((1-\lambda) g(a)+\lambda g(b))+\lambda f(a)+(1-\lambda) f(b)\right] \\
& \leq \frac{f(a)+f(b)}{2}
\end{aligned}
$$

for any $\lambda \in[0,1]$. 
Proof. From the inequality (2.1) we have for the convex function $f \circ g^{-1}$ and $c$, $d \in[g(a), g(b)]$ that

$$
\begin{aligned}
& f \circ g^{-1}\left(\frac{c+d}{2}\right) \\
& \leq \lambda f \circ g^{-1}\left(\frac{\lambda d+(2-\lambda) c}{2}\right)+(1-\lambda) f \circ g^{-1}\left(\frac{(1+\lambda) d+(1-\lambda) c}{2}\right) \\
& \leq \frac{1}{d-c} \int_{c}^{d} f \circ g^{-1}(u) d u \\
& \leq \frac{1}{2}\left[f \circ g^{-1}((1-\lambda) c+\lambda d)+\lambda f \circ g^{-1}(c)+(1-\lambda) f \circ g^{-1}(d)\right] \\
& \leq \frac{f \circ g^{-1}(c)+f \circ g^{-1}(d)}{2}
\end{aligned}
$$

for any $\lambda \in[0,1]$.

If we take $c=g(a)$ and $d=g(b)$, then we get

$$
\begin{aligned}
& f \circ g^{-1}\left(\frac{g(a)+g(b)}{2}\right) \\
& \leq \lambda f \circ g^{-1}\left(\frac{\lambda g(b)+(2-\lambda) g(a)}{2}\right) \\
& +(1-\lambda) f \circ g^{-1}\left(\frac{(1+\lambda) g(b)+(1-\lambda) g(a)}{2}\right) \\
& \leq \frac{1}{g(b)-g(a)} \int_{g(a)}^{g(b)} f \circ g^{-1}(u) d u \\
& \leq \frac{1}{2}\left[f \circ g^{-1}((1-\lambda) g(a)+\lambda g(b))+\lambda f(a)+(1-\lambda) f(b)\right] \\
& \leq \frac{f(a)+f(b)}{2}
\end{aligned}
$$

for any $\lambda \in[0,1]$.

Using the change of variable $g^{-1}(u)=t, t \in[a, b]$ we have $u=g(t), d u=g^{\prime}(t) d t$ and

$$
\int_{g(a)}^{g(b)} f \circ g^{-1}(u) d u=\int_{a}^{b} f(t) g^{\prime}(t) d t
$$

and by (2.8) we get the desired result (2.6).

Corollary 1. With the assumptions of Theorem 1 we have

$$
\begin{aligned}
f\left(M_{g}(a, b)\right) & \leq \frac{1}{2}\left[f \circ g^{-1}\left(\frac{g(b)+3 g(a)}{4}\right)+f \circ g^{-1}\left(\frac{g(a)+3 g(b)}{4}\right)\right] \\
& \leq \frac{1}{g(b)-g(a)} \int_{a}^{b} f(t) g^{\prime}(t) d t \\
& \leq \frac{1}{2}\left[f\left(M_{g}(a, b)\right)+\frac{f(a)+f(b)}{2}\right] \leq \frac{f(a)+f(b)}{2} .
\end{aligned}
$$


Remark 1. Using the change of variable $u=(1-s) c+s d, s \in[0,1]$, then we have $d u=(d-c) d s$, which gives that

$$
\frac{1}{d-c} \int_{c}^{d} h(u) d u=\int_{0}^{1} h((1-s) c+s d) d s
$$

Using this fact, we have from Theorem 1 the following inequality

$$
\begin{aligned}
f\left(M_{g}(a, b)\right) & \leq \lambda f \circ g^{-1}\left(\frac{\lambda g(b)+(2-\lambda) g(a)}{2}\right) \\
& +(1-\lambda) f \circ g^{-1}\left(\frac{(1+\lambda) g(b)+(1-\lambda) g(a)}{2}\right) \\
& \leq \frac{b-a}{g(b)-g(a)} \int_{0}^{1} f((1-s) a+s b) g^{\prime}((1-s) a+s b) d s \\
& =\int_{0}^{1} f \circ g^{-1}((1-\tau) g(a)+\tau g(b)) d \tau \\
& \leq \frac{1}{2}\left[f \circ g^{-1}((1-\lambda) g(a)+\lambda g(b))+\lambda f(a)+(1-\lambda) f(b)\right] \\
& \leq \frac{f(a)+f(b)}{2}
\end{aligned}
$$

for all $\lambda \in[0,1]$.

Corollary 2. Let $g:[a, b] \rightarrow[g(a), g(b)]$ be a continuous strictly increasing function that is differentiable on $(a, b), f:[a, b] \rightarrow J, J$ an interval of real numbers and $k: J \rightarrow \mathbb{R}$ a continuous function on $J$ that is strictly increasing (decreasing) on $J$. If the function $f:[a, b] \rightarrow J$ is $k$-composite- $g^{-1}$ convex on $[a, b]$, then

$$
\begin{gathered}
\text { (2.11) } f\left(M_{g}(a, b)\right) \\
\leq(\geq) k^{-1}\left\{\lambda k \circ f \circ g^{-1}\left(\frac{\lambda g(b)+(2-\lambda) g(a)}{2}\right)\right. \\
\left.+(1-\lambda) k \circ f \circ g^{-1}\left(\frac{(1+\lambda) g(b)+(1-\lambda) g(a)}{2}\right)\right\} \\
\leq(\geq) k^{-1}\left(\frac{1}{g(b)-g(a)} \int_{a}^{b} k \circ f(t) g^{\prime}(t) d t\right) \\
\leq(\geq) k^{-1}\left\{\frac{1}{2}\left[k \circ f \circ g^{-1}((1-\lambda) g(a)+\lambda g(b))+\lambda k \circ f(a)+(1-\lambda) k \circ f(b)\right]\right\} \\
\leq(\geq) k^{-1}\left(\frac{k \circ f(a)+k \circ f(b)}{2}\right)
\end{gathered}
$$

for any $\lambda \in[0,1]$. 
Proof. From (2.6) we have

$$
\begin{aligned}
& k \circ f\left(M_{g}(a, b)\right) \\
& \leq \lambda k \circ f \circ g^{-1}\left(\frac{\lambda g(b)+(2-\lambda) g(a)}{2}\right) \\
& +(1-\lambda) k \circ f \circ g^{-1}\left(\frac{(1+\lambda) g(b)+(1-\lambda) g(a)}{2}\right) \\
& \leq \frac{1}{g(b)-g(a)} \int_{a}^{b} k \circ f(t) g^{\prime}(t) d t \\
& \leq \frac{1}{2}\left[k \circ f \circ g^{-1}((1-\lambda) g(a)+\lambda g(b))+\lambda k \circ f(a)+(1-\lambda) k \circ f(b)\right] \\
& \leq \frac{k \circ f(a)+k \circ f(b)}{2}
\end{aligned}
$$

for any $\lambda \in[0,1]$.

Taking $k^{-1}$ in (2.12) we obtain the desired result (2.11).

In 1906, Fejér [17], while studying trigonometric polynomials, obtained the following inequalities which generalize that of Hermite \& Hadamard:

Theorem 2 (Fejér's Inequality). Consider the integral $\int_{a}^{b} h(x) w(x) d x$, where $h$ is a convex function in the interval $(a, b)$ and $w$ is a positive function in the same interval such that

$$
w(x)=w(a+b-x), \text { for any } x \in[a, b]
$$

i.e., $y=w(x)$ is a symmetric curve with respect to the straight line which contains the point $\left(\frac{1}{2}(a+b), 0\right)$ and is normal to the $x$-axis. Under those conditions the following inequalities are valid:

$$
h\left(\frac{a+b}{2}\right) \int_{a}^{b} w(x) d x \leq \int_{a}^{b} h(x) w(x) d x \leq \frac{h(a)+h(b)}{2} \int_{a}^{b} w(x) d x
$$

If $h$ is concave on $(a, b)$, then the inequalities reverse in (2.13).

If $w:[a, b] \rightarrow \mathbb{R}$ is continuous and positive on the interval $[a, b]$, then the function $W:[a, b] \rightarrow[0, \infty)$ is strictly increasing and differentiable on $(a, b)$ and the inverse $W^{-1}:\left[a, \int_{a}^{b} w(s) d s\right] \rightarrow[a, b]$ exists.

Corollary 3. Assume that $w:[a, b] \rightarrow \mathbb{R}$ is continuous and positive on the interval $[a, b]$ and $f:[a, b] \rightarrow \mathbb{R}$ is composite- $W^{-1}$ convex on $[a, b]$, then we have the 
following Fejér's type inequality

$$
\begin{aligned}
& f\left[W^{-1}\left(\frac{1}{2} \int_{a}^{b} w(s) d s\right)\right] \\
& \leq \lambda f\left[W^{-1}\left(\frac{1}{2} \lambda \int_{a}^{b} w(s) d s\right)\right]+(1-\lambda) f\left[W^{-1}\left(\frac{1}{2}(1+\lambda) \int_{a}^{b} w(s) d s\right)\right] \\
& \leq \frac{1}{\int_{a}^{b} w(s)} \int_{a}^{b} f(t) w(t) d t \\
& \leq \frac{1}{2}\left[f\left[W^{-1}\left(\lambda \int_{a}^{b} w(s) d s\right)\right]+\lambda f(a)+(1-\lambda) f(b)\right] \leq \frac{f(a)+f(b)}{2}
\end{aligned}
$$

for all $\lambda \in[0,1]$.

In particular, we have

$$
\begin{aligned}
& f\left[W^{-1}\left(\frac{1}{2} \int_{a}^{b} w(s) d s\right)\right] \\
& \leq \frac{1}{2} f\left[W^{-1}\left(\frac{1}{4} \int_{a}^{b} w(s) d s\right)\right]+\frac{1}{2} f\left[W^{-1}\left(\frac{3}{4} \int_{a}^{b} w(s) d s\right)\right] \\
& \quad \leq \frac{1}{\int_{a}^{b} w(s)} \int_{a}^{b} f(t) w(t) d t \\
& \leq \frac{1}{2}\left[f\left[W^{-1}\left(\frac{1}{2} \int_{a}^{b} w(s) d s\right)\right]+\frac{f(a)+f(b)}{2}\right] \leq \frac{f(a)+f(b)}{2} .
\end{aligned}
$$

Remark 2. Assume that $w:[a, b] \rightarrow \mathbb{R}$ is continuous and positive on the interval $[a, b], f:[a, b] \rightarrow J, J$ an interval of real numbers and $k: J \rightarrow \mathbb{R}$ a continuous function on $J$ that is strictly increasing (decreasing) on $J$. If the function $f:[a, b] \rightarrow$ $J$ is $k$-composite- $W^{-1}$ convex on $[a, b]$, then

$$
\begin{gathered}
\text { 2.16) } f\left[W^{-1}\left(\frac{1}{2} \int_{a}^{b} w(s) d s\right)\right] \\
\leq(\geq) k^{-1}\left\{\lambda k \circ f\left[W^{-1}\left(\frac{1}{2} \lambda \int_{a}^{b} w(s) d s\right)\right]\right. \\
\left.+(1-\lambda) k \circ f\left[W^{-1}\left(\frac{1}{2}(1+\lambda) \int_{a}^{b} w(s) d s\right)\right]\right\} \\
\leq(\geq) k^{-1}\left(\frac{1}{\int_{a}^{b} w(s)} \int_{a}^{b} k \circ f(t) w(t) d t\right) \\
\leq(\geq) k^{-1}\left\{\frac{1}{2}\left[k \circ f\left[W^{-1}\left(\lambda \int_{a}^{b} w(s) d s\right)\right]+\lambda k \circ f(a)+(1-\lambda) k \circ f(b)\right]\right\} \\
\leq(\geq) k^{-1}\left(\frac{k \circ f(a)+k \circ f(b)}{2}\right)
\end{gathered}
$$

for all $\lambda \in[0,1]$. 
In particular, we have

$$
\begin{aligned}
& \text { (2.17) } f\left[W^{-1}\left(\frac{1}{2} \int_{a}^{b} w(s) d s\right)\right] \\
& \leq(\geq) k^{-1}\left\{\frac{1}{2} k \circ f\left[W^{-1}\left(\frac{1}{4} \int_{a}^{b} w(s) d s\right)\right]+\frac{1}{2} k \circ f\left[W^{-1}\left(\frac{3}{4} \int_{a}^{b} w(s) d s\right)\right]\right\} \\
& \leq(\geq) k^{-1}\left(\frac{1}{\int_{a}^{b} w(s)} \int_{a}^{b} k \circ f(t) w(t) d t\right) \\
& \leq(\geq) k^{-1}\left\{\frac{1}{2}\left[k \circ f\left[W^{-1}\left(\frac{1}{2} \int_{a}^{b} w(s) d s\right)\right]+\frac{1}{2} k \circ f(a)+\frac{1}{2} k \circ f(b)\right]\right\} \\
& \leq(\geq) k^{-1}\left(\frac{k \circ f(a)+k \circ f(b)}{2}\right)
\end{aligned}
$$

\section{Reverse Inequalities}

The following reverse inequalities may be stated:

Theorem 3. Let $g:[a, b] \rightarrow[g(a), g(b)]$ be a continuous strictly increasing function that is differentiable on $(a, b)$. If $f:[a, b] \rightarrow \mathbb{R}$ is composite-g ${ }^{-1}$ convex on $[a, b]$, then

$$
\begin{aligned}
0 & \leq \frac{1}{g(b)-g(a)} \int_{a}^{b} f(t) g^{\prime}(t) d t-f\left(M_{g}(a, b)\right) \\
& \leq \frac{1}{8}(g(b)-g(a))\left[\frac{f_{-}^{\prime}(b)}{g_{-}^{\prime}(b)}-\frac{f_{+}^{\prime}(a)}{g_{+}^{\prime}(a)}\right]
\end{aligned}
$$

and

$$
\begin{aligned}
0 & \leq \frac{f(a)+f(b)}{2}-\frac{1}{g(b)-g(a)} \int_{a}^{b} f(t) g^{\prime}(t) d t \\
& \leq \frac{1}{8}(g(b)-g(a))\left[\frac{f_{-}^{\prime}(b)}{g_{-}^{\prime}(b)}-\frac{f_{+}^{\prime}(a)}{g_{+}^{\prime}(a)}\right],
\end{aligned}
$$

provided that the lateral derivatives $f_{+}^{\prime}(a), g_{+}^{\prime}(a), f_{-}^{\prime}(b)$ and $g_{-}^{\prime}(b)$ are finite.

Proof. Let $h:[c, d] \rightarrow \mathbb{R}$ be a convex function on $[c, d]$. We use the inequality that has been established in [4]

$$
0 \leq \frac{1}{d-c} \int_{c}^{d} h(u) d u-h\left(\frac{c+d}{2}\right) \leq \frac{1}{8}(d-c)\left[h_{-}^{\prime}(d)-h_{+}^{\prime}(c)\right]
$$

and the inequality obtained in [5]

$$
0 \leq \frac{h(c)+h(d)}{2}-\frac{1}{d-c} \int_{c}^{d} h(u) d u \leq \frac{1}{8}(d-c)\left[h_{-}^{\prime}(d)-h_{+}^{\prime}(c)\right] .
$$

The constant $\frac{1}{8}$ is best possible in both (3.3) and (3.4). 
From the inequalities (3.3) and (3.4) we have for the convex function $h=f \circ g^{-1}$ and $c, d \in[g(a), g(b)]$ that

$$
\begin{aligned}
0 & \leq \frac{1}{d-c} \int_{c}^{d}\left(f \circ g^{-1}\right)(u) d u-\left(f \circ g^{-1}\right)\left(\frac{c+d}{2}\right) \\
& \leq \frac{1}{8}(d-c)\left[\left(f \circ g^{-1}\right)_{-}^{\prime}(d)-\left(f \circ g^{-1}\right)_{+}^{\prime}(c)\right]
\end{aligned}
$$

and

$$
\begin{aligned}
0 & \leq \frac{\left(f \circ g^{-1}\right)(c)+\left(f \circ g^{-1}\right)(d)}{2}-\frac{1}{d-c} \int_{c}^{d}\left(f \circ g^{-1}\right)(u) d u \\
& \leq \frac{1}{8}(d-c)\left[\left(f \circ g^{-1}\right)_{-}^{\prime}(d)-\left(f \circ g^{-1}\right)_{+}^{\prime}(c)\right] .
\end{aligned}
$$

Since $f \circ g^{-1}$ has lateral derivatives for $z \in(g(a), g(b))$ it follows $f$ has lateral derivatives in each point of $(a, b)$ and by the chain rule and the derivative of the inverse function, we have

$$
\left(f \circ g^{-1}\right)_{ \pm}^{\prime}(z)=\left(f_{ \pm}^{\prime} \circ g^{-1}\right)(z)\left(g^{-1}\right)^{\prime}(z)=\frac{\left(f_{ \pm}^{\prime} \circ g^{-1}\right)(z)}{\left(g^{\prime} \circ g^{-1}\right)(z)} .
$$

Therefore, by (3.5) and (3.6) we get

$$
\begin{aligned}
0 & \leq \frac{1}{d-c} \int_{c}^{d}\left(f \circ g^{-1}\right)(u) d u-\left(f \circ g^{-1}\right)\left(\frac{c+d}{2}\right) \\
& \leq \frac{1}{8}(d-c)\left[\frac{\left(f_{-}^{\prime} \circ g^{-1}\right)(d)}{\left(g^{\prime} \circ g^{-1}\right)(d)}-\frac{\left(f_{+}^{\prime} \circ g^{-1}\right)(c)}{\left(g^{\prime} \circ g^{-1}\right)(c)}\right]
\end{aligned}
$$

and

$$
\begin{aligned}
0 & \leq \frac{\left(f \circ g^{-1}\right)(c)+\left(f \circ g^{-1}\right)(d)}{2}-\frac{1}{d-c} \int_{c}^{d}\left(f \circ g^{-1}\right)(u) d u \\
& \leq \frac{1}{8}(d-c)\left[\frac{\left(f_{-}^{\prime} \circ g^{-1}\right)(d)}{\left(g^{\prime} \circ g^{-1}\right)(d)}-\frac{\left(f_{+}^{\prime} \circ g^{-1}\right)(c)}{\left(g^{\prime} \circ g^{-1}\right)(c)}\right]
\end{aligned}
$$

and by taking $c=g(a)$ and $d=g(b)$ in (3.8) and (3.9), then we get the desired results (3.1) and (3.2).

Corollary 4. Assume that $w:[a, b] \rightarrow \mathbb{R}$ is continuous and positive on the interval $[a, b]$. If $f:[a, b] \rightarrow \mathbb{R}$ is composite- $W^{-1}$ convex on $[a, b]$, then we have the following weighted reverse integral inequalities

$$
\begin{aligned}
0 & \leq \frac{1}{\int_{a}^{b} w(s)} \int_{a}^{b} f(t) w(t) d t-f\left[W^{-1}\left(\frac{1}{2} \int_{a}^{b} w(s) d s\right)\right] \\
& \leq \frac{1}{8}\left[\frac{f_{-}^{\prime}(b)}{w(b)}-\frac{f_{+}^{\prime}(a)}{w(a)}\right] \int_{a}^{b} w(s) d s
\end{aligned}
$$

and

$$
\begin{aligned}
0 & \leq \frac{f(a)+f(b)}{2}-\frac{1}{\int_{a}^{b} w(s)} \int_{a}^{b} f(t) w(t) d t \\
& \leq \frac{1}{8}\left[\frac{f_{-}^{\prime}(b)}{w(b)}-\frac{f_{+}^{\prime}(a)}{w(a)}\right] \int_{a}^{b} w(s) d s
\end{aligned}
$$


provided that $f_{-}^{\prime}(b)$ and $f_{+}^{\prime}(a)$ are finite.

Remark 3. Let $g:[a, b] \rightarrow[g(a), g(b)]$ be a continuous strictly increasing function that is differentiable on $(a, b), f:[a, b] \rightarrow J, J$ an interval of real numbers and $k:$ $J \rightarrow \mathbb{R}$ a continuous function on $J$ that is strictly increasing on $J$ and differentiable on the interior of $J$. If the function $f:[a, b] \rightarrow J$ is $k$-composite- $g^{-1}$ convex on $[a, b]$ and $f_{+}^{\prime}(a), g_{+}^{\prime}(a), f_{-}^{\prime}(b), g_{-}^{\prime}(b), k^{\prime}(f(a))$ and $k^{\prime}(f(b))$ are finite, then by Theorem 3 we have

$$
\begin{aligned}
0 & \leq \frac{1}{g(b)-g(a)} \int_{a}^{b}(k \circ f)(t) g^{\prime}(t) d t-k \circ f\left(M_{g}(a, b)\right) \\
& \leq \frac{1}{8}(g(b)-g(a))\left[\frac{k^{\prime}(f(b)) f_{-}^{\prime}(b)}{g_{-}^{\prime}(b)}-\frac{k^{\prime}(f(a)) f_{+}^{\prime}(a)}{g_{+}^{\prime}(a)}\right]
\end{aligned}
$$

and

$$
\begin{aligned}
0 & \leq \frac{k \circ f(a)+k \circ f(b)}{2}-\frac{1}{g(b)-g(a)} \int_{a}^{b}(k \circ f)(t) g^{\prime}(t) d t \\
& \leq \frac{1}{8}(g(b)-g(a))\left[\frac{k^{\prime}(f(b)) f_{-}^{\prime}(b)}{g_{-}^{\prime}(b)}-\frac{k^{\prime}(f(a)) f_{+}^{\prime}(a)}{g_{+}^{\prime}(a)}\right] .
\end{aligned}
$$

Assume that $w:[a, b] \rightarrow \mathbb{R}$ is continuous and positive on the interval $[a, b]$, $f:[a, b] \rightarrow J, J$ an interval of real numbers and $k: J \rightarrow \mathbb{R}$ a continuous function on $J$ that is strictly increasing on $J$ and differentiable on the interior of $J$. If the function $f:[a, b] \rightarrow J$ is $k$-composite- $W^{-1}$ convex on $[a, b]$ and $f_{+}^{\prime}(a), f_{-}^{\prime}(b)$, $k^{\prime}(f(a))$ and $k^{\prime}(f(b))$ are finite, then we have the weighted inequalities

$$
\begin{aligned}
0 & \leq \frac{1}{g(b)-g(a)} \int_{a}^{b}(k \circ f)(t) w(t) d t-k \circ f\left(W^{-1}\left(\frac{1}{2} \int_{a}^{b} w(s) d s\right)\right) \\
& \leq \frac{1}{8}(g(b)-g(a))\left[\frac{k^{\prime}(f(b)) f_{-}^{\prime}(b)}{w(b)}-\frac{k^{\prime}(f(a)) f_{+}^{\prime}(a)}{w(a)}\right]
\end{aligned}
$$

and

$$
\begin{aligned}
0 & \leq \frac{k \circ f(a)+k \circ f(b)}{2}-\frac{1}{g(b)-g(a)} \int_{a}^{b}(k \circ f)(t) w(t) d t \\
& \leq \frac{1}{8}(g(b)-g(a))\left[\frac{k^{\prime}(f(b)) f_{-}^{\prime}(b)}{w(b)}-\frac{k^{\prime}(f(a)) f_{+}^{\prime}(a)}{w(a)}\right] .
\end{aligned}
$$

\section{Applications for $A G$ and $A H$-Convex Functions}

The function $f:[a, b] \rightarrow(0, \infty)$ is $A G$-convex means that $f$ is $k$-composite convex on $[a, b]$ with $k(t)=\ln t, t>0$. By making use of Corollary 2 for $g(t)=t$, we get

$$
\begin{aligned}
f\left(\frac{a+b}{2}\right) \leq f^{\lambda}\left(\frac{\lambda b+(2-\lambda) a}{2}\right) f^{1-\lambda}\left(\frac{(1+\lambda) b+(1-\lambda) a}{2}\right) \\
\quad \leq \exp \left(\frac{1}{b-a} \int_{a}^{b} \ln f(t) d t\right) \\
\leq \sqrt{f((1-\lambda) a+\lambda b) f^{\lambda}(a) f^{1-\lambda}(b)} \leq \sqrt{f(a) f(b)}
\end{aligned}
$$

for any $\lambda \in[0,1]$, see also $[9]$. 
If we use Remark 3 for $g(t)=t$, then we get

$$
0 \leq \frac{1}{b-a} \int_{a}^{b} \ln f(t) d t-\ln f\left(\frac{a+b}{2}\right) \leq \frac{1}{8}(b-a)\left[\frac{f_{-}^{\prime}(b)}{f(b)}-\frac{f_{+}^{\prime}(a)}{f(a)}\right]
$$

and

$$
0 \leq \frac{\ln f(a)+\ln f(b)}{2}-\frac{1}{b-a} \int_{a}^{b} \ln f(t) d t \leq \frac{1}{8}(b-a)\left[\frac{f_{-}^{\prime}(b)}{f(b)}-\frac{f_{+}^{\prime}(a)}{f(a)}\right]
$$

By taking the exponential in (4.2) and (4.3) we get the equivalent inequalities

$$
1 \leq \frac{\exp \left(\frac{1}{b-a} \int_{a}^{b} \ln f(t) d t\right)}{f\left(\frac{a+b}{2}\right)} \leq \exp \left\{\frac{1}{8}(b-a)\left[\frac{f_{-}^{\prime}(b)}{f(b)}-\frac{f_{+}^{\prime}(a)}{f(a)}\right]\right\}
$$

and

$$
1 \leq \frac{\sqrt{f(a) f(b)}}{\exp \left(\frac{1}{b-a} \int_{a}^{b} \ln f(t) d t\right)} \leq \exp \left\{\frac{1}{8}(b-a)\left[\frac{f_{-}^{\prime}(b)}{f(b)}-\frac{f_{+}^{\prime}(a)}{f(a)}\right]\right\}
$$

that was obtained in [9].

The function $f:[a, b] \rightarrow(0, \infty)$ is $A H$-convex on $[a, b]$ means that $f$ is $k$ composite concave on $[a, b]$ with $k:(0, \infty) \rightarrow(0, \infty), k(t)=\frac{1}{t}$. By making use of Corollary 2 for $g(t)=t$, we get

$$
\begin{gathered}
f\left(\frac{a+b}{2}\right) \\
\leq\left\{\lambda f^{-1}\left(\frac{\lambda b+(2-\lambda) a}{2}\right)+(1-\lambda) f^{-1}\left(\frac{(1+\lambda) b+(1-\lambda) a}{2}\right)\right\}^{-1} \\
\leq\left(\frac{1}{b-a} \int_{a}^{b} f^{-1}(t) d t\right)^{-1} \\
\leq\left\{\frac{1}{2}\left[f^{-1}((1-\lambda) a+\lambda b)+\lambda f^{-1}(a)+(1-\lambda) f^{-1}(b)\right]\right\}^{-1} \\
\leq\left(\frac{f^{-1}(a)+f^{-1}(b)}{2}\right)^{-1}
\end{gathered}
$$

for any $\lambda \in[0,1]$.

By taking the power -1 , this inequality is equivalent to

$$
\begin{aligned}
& f^{-1}\left(\frac{a+b}{2}\right) \\
& \geq \lambda f^{-1}\left(\frac{\lambda b+(2-\lambda) a}{2}\right)+(1-\lambda) f^{-1}\left(\frac{(1+\lambda) b+(1-\lambda) a}{2}\right) \\
& \geq \frac{1}{b-a} \int_{a}^{b} f^{-1}(t) d t \\
& \geq \frac{1}{2}\left[f^{-1}((1-\lambda) a+\lambda b)+\lambda f^{-1}(a)+(1-\lambda) f^{-1}(b)\right] \geq \frac{f^{-1}(a)+f^{-1}(b)}{2}
\end{aligned}
$$

for any $\lambda \in[0,1]$. 
If we use Remark 3 for $g(t)=t$, then we get

$$
0 \leq f^{-1}\left(\frac{a+b}{2}\right)-\frac{1}{b-a} \int_{a}^{b} f^{-1}(t) d t \leq \frac{1}{8}(b-a)\left[\frac{f_{-}^{\prime}(b)}{f^{2}(b)}-\frac{f_{+}^{\prime}(a)}{f^{2}(a)}\right]
$$

and

$$
0 \leq \frac{1}{b-a} \int_{a}^{b} f^{-1}(t) d t-\frac{f^{-1}(a)+f^{-1}(b)}{2} \leq \frac{1}{8}(b-a)\left[\frac{f_{-}^{\prime}(b)}{f^{2}(b)}-\frac{f_{+}^{\prime}(a)}{f^{2}(a)}\right]
$$

\section{Applications for $G A, G G$ and $G H$-Convex Functions}

If we take $g(t)=\ln t, t \in[a, b] \subset(0, \infty)$, then $f:[a, b] \rightarrow \mathbb{R}$ is $G A$-convex on $[a, b]$ means that that $f:[a, b] \rightarrow \mathbb{R}$ composite- $g^{-1}$ convex on $[a, b]$. By making use of Corollary 2 for $k(t)=t$, we get

$$
\begin{aligned}
f(\sqrt{a b}) & \leq(1-\lambda) f\left(a^{\frac{1-\lambda}{2}} b^{\frac{\lambda+1}{2}}\right)+\lambda f\left(a^{\frac{2-\lambda}{2}} b^{\frac{\lambda}{2}}\right) \\
& \leq \frac{1}{\ln \left(\frac{b}{a}\right)} \int_{a}^{b} \frac{f(t)}{t} d t \\
& \leq \frac{1}{2}\left[f\left(a^{1-\lambda} b^{\lambda}\right)+(1-\lambda) f(b)+\lambda f(a)\right] \leq \frac{f(a)+f(b)}{2}
\end{aligned}
$$

for any $\lambda \in[0,1]$. This result was obtained in [10].

If we use Remark 3 for $k(t)=t$, then we get

$$
0 \leq \frac{1}{\ln \left(\frac{b}{a}\right)} \int_{a}^{b} \frac{f(t)}{t} d t-f(\sqrt{a b}) \leq \frac{1}{8} \ln \left(\frac{b}{a}\right)\left[b f_{-}^{\prime}(b)-a f_{+}^{\prime}(a)\right]
$$

and

$$
0 \leq \frac{f(a)+f(b)}{2}-\frac{1}{\ln \left(\frac{b}{a}\right)} \int_{a}^{b} \frac{f(t)}{t} d t \leq \frac{1}{8} \ln \left(\frac{b}{a}\right)\left[b f_{-}^{\prime}(b)-a f_{+}^{\prime}(a)\right] .
$$

These results were also obtained in [10].

The function $f: I \subset(0, \infty) \rightarrow(0, \infty)$ is $G G$-convex means that $f$ is $k$-composite$g^{-1}$ convex on $[a, b]$ with $k:(0, \infty) \rightarrow \mathbb{R}, k(t)=\ln t$ and $g(t)=\ln t, t \in[a, b]$. By making use of Corollary 2 we get

$$
\begin{aligned}
f(\sqrt{a b}) \leq f^{\lambda}\left(a^{\frac{2-\lambda}{2}} b^{\frac{\lambda}{2}}\right) f^{1-\lambda}\left(a^{\frac{1-\lambda}{2}} b^{\frac{\lambda+1}{2}}\right) \\
\leq \exp \left(\frac{1}{\ln \left(\frac{b}{a}\right)} \int_{a}^{b} \frac{\ln f(t)}{t} d t\right) \\
\leq \sqrt{f\left(a^{1-\lambda} b^{\lambda}\right) f^{\lambda}(a) f^{1-\lambda}(b)} \leq \sqrt{f(a) f(b)}
\end{aligned}
$$

for any $\lambda \in[0,1]$. This result was obtained in [11], see also [12].

If we use Remark 3 , then we have the inequalities

$$
1 \leq \frac{\sqrt{f(a) f(b)}}{\exp \left(\frac{1}{\ln b-\ln a} \int_{a}^{b} \frac{\ln f(s)}{s} d s\right)} \leq\left(\frac{b}{a}\right)^{\frac{1}{8}\left[\frac{f_{-}^{\prime}(b) b}{f(b)}-\frac{f_{+}^{\prime}(a) a}{f(a)}\right]}
$$


and

$$
1 \leq \frac{\exp \left(\frac{1}{\ln b-\ln a} \int_{a}^{b} \frac{\ln f(s)}{s} d s\right)}{f(\sqrt{a b})} \leq\left(\frac{b}{a}\right)^{\frac{1}{8}\left[\frac{f_{-}^{\prime}(b) b}{f(b)}-\frac{f_{+}^{\prime}(a) a}{f(a)}\right]} .
$$

These results were obtained in [11], see also [12].

We also have that $f:[a, b] \subset(0, \infty) \rightarrow \mathbb{R}$ is $G H$-convex on $[a, b]$ is equivalent to the fact that $f$ is $k$-composite- $g^{-1}$ concave on $[a, b]$ with $k:(0, \infty) \rightarrow(0, \infty)$, $k(t)=\frac{1}{t}$ and $g(t)=\ln t, t \in I$. By making use of Corollary 2 we get

$$
\begin{aligned}
& f(\sqrt{a b}) \leq\left[\lambda f^{-1}\left(a^{\frac{2-\lambda}{2}} b^{\frac{\lambda}{2}}\right)+(1-\lambda) f^{-1}\left(a^{\frac{1-\lambda}{2}} b^{\frac{\lambda+1}{2}}\right)\right]^{-1} \\
& \leq\left(\frac{1}{\ln \left(\frac{b}{a}\right)} \int_{a}^{b} \frac{f^{-1}(t)}{t} d t\right)^{-1} \\
& \leq\left\{\frac{1}{2}\left[f^{-1}\left(a^{1-\lambda} b^{\lambda}\right)+\lambda f^{-1}(a)+(1-\lambda) f^{-1}(b)\right]\right\}^{-1} \\
& \leq\left(\frac{f^{-1}(a)+f^{-1}(b)}{2}\right)^{-1}
\end{aligned}
$$

for any $\lambda \in[0,1]$.

This is equivalent to

$$
\begin{aligned}
& f^{-1}(\sqrt{a b}) \geq \lambda f^{-1}\left(a^{\frac{2-\lambda}{2}} b^{\frac{\lambda}{2}}\right)+(1-\lambda) f^{-1}\left(a^{\frac{1-\lambda}{2}} b^{\frac{\lambda+1}{2}}\right) \\
& \geq \frac{1}{\ln \left(\frac{b}{a}\right)} \int_{a}^{b} \frac{f^{-1}(t)}{t} d t \\
& \geq \frac{1}{2}\left[f^{-1}\left(a^{1-\lambda} b^{\lambda}\right)+\lambda f^{-1}(a)+(1-\lambda) f^{-1}(b)\right] \\
& \quad \geq \frac{f^{-1}(a)+f^{-1}(b)}{2} .
\end{aligned}
$$

If we use Remark 3, then we get

$$
0 \leq f^{-1}(\sqrt{a b})-\frac{1}{\ln \left(\frac{b}{a}\right)} \int_{a}^{b} \frac{f^{-1}(t)}{t} d t \leq \frac{1}{8} \ln \left(\frac{b}{a}\right)\left[\frac{b f_{-}^{\prime}(b)}{f^{2}(b)}-\frac{a f_{+}^{\prime}(a)}{f^{2}(a)}\right]
$$

and

$$
\begin{aligned}
0 \leq \frac{1}{\ln \left(\frac{b}{a}\right)} \int_{a}^{b} \frac{f^{-1}(t)}{t} d t-\frac{f^{-1}(a)+f^{-1}(b)}{2} & \\
& \leq \frac{1}{8} \ln \left(\frac{b}{a}\right)\left[\frac{b f_{-}^{\prime}(b)}{f^{2}(b)}-\frac{a f_{+}^{\prime}(a)}{f^{2}(a)}\right]
\end{aligned}
$$

\section{Applications for $H A, H G$ and $H H$-Convex Functions}

Let $f:[a, b] \subset(0, \infty) \rightarrow \mathbb{R}$ be an $H A$-convex function on the interval $[a, b]$. This is equivalent to the fact that $f$ is composite- $g^{-1}$ convex on $[a, b]$ with the increasing function $g(t)=-\frac{1}{t}$. Then by applying Corollary 2 for $k(t)=t$, we have 
the inequalities

$$
\begin{aligned}
f\left(\frac{2 a b}{a+b}\right) & \leq(1-\lambda) f\left(\frac{2 a b}{(1-\lambda) a+(\lambda+1) b}\right)+\lambda f\left(\frac{2 a b}{(2-\lambda) a+\lambda b}\right) \\
& \leq \frac{a b}{b-a} \int_{a}^{b} \frac{f(t)}{t^{2}} d t \\
& \leq \frac{1}{2}\left[f\left(\frac{a b}{(1-\lambda) a+\lambda b}\right)+(1-\lambda) f(a)+\lambda f(b)\right] \\
& \leq \frac{f(a)+f(b)}{2}
\end{aligned}
$$

for any $\lambda \in[0,1]$. This result was obtained in [13].

If we use Remark 3 , then we get

$$
0 \leq \frac{a b}{b-a} \int_{a}^{b} \frac{f(t)}{t^{2}} d t-f\left(\frac{2 a b}{a+b}\right) \leq \frac{1}{8}\left[\frac{f_{-}^{\prime}(b) b^{2}-f_{+}^{\prime}(a) a^{2}}{a b}\right](b-a)
$$

and

$$
0 \leq \frac{f(a)+f(b)}{2}-\frac{a b}{b-a} \int_{a}^{b} \frac{f(t)}{t^{2}} d t \leq \frac{1}{8}\left[\frac{f_{-}^{\prime}(b) b^{2}-f_{+}^{\prime}(a) a^{2}}{a b}\right](b-a) .
$$

This results were obtained in [13].

Let $f:[a, b] \subset(0, \infty) \rightarrow(0, \infty)$ be an $H G$-convex function on the interval $[a, b]$. This is equivalent to the fact that $f$ is $k$-composite- $g^{-1}$ concave on $[a, b]$ with $k:(0, \infty) \rightarrow(0, \infty), k(t)=\frac{1}{t}$ and $g(t)=\ln t, t \in[a, b]$. Then by applying Corollary 2 , we have the inequalities

$$
\begin{aligned}
f\left(\frac{2 a b}{a+b}\right) & \leq f^{1-\lambda}\left(\frac{2 a b}{(1-\lambda) a+(\lambda+1) b}\right) f^{\lambda}\left(\frac{2 a b}{(2-\lambda) a+\lambda b}\right) \\
& \leq \exp \left(\frac{a b}{b-a} \int_{a}^{b} \frac{\ln f(t)}{t^{2}} d t\right) \\
& \leq \sqrt{f\left(\frac{a b}{(1-\lambda) a+\lambda b}\right)[f(a)]^{1-\lambda}[f(b)]^{\lambda}} \leq \sqrt{f(a) f(b)}
\end{aligned}
$$

for any $\lambda \in[0,1]$. This result was obtained in [14].

If we use Remark 3 , then we get

$$
1 \leq \frac{\exp \left(\frac{a b}{b-a} \int_{a}^{b} \frac{\ln f(t)}{t^{2}} d t\right)}{f\left(\frac{2 a b}{a+b}\right)} \leq \exp \left(\frac{1}{8}\left[\frac{f_{-}^{\prime}(b) b^{2}}{f(b)}-\frac{f_{+}^{\prime}(a) a^{2}}{f(a)}\right] \frac{b-a}{a b}\right)
$$

and

$$
1 \leq \frac{\sqrt{f(a) f(b)}}{\exp \left(\frac{a b}{b-a} \int_{a}^{b} \frac{\ln f(t)}{t^{2}} d t\right)} \leq \exp \left(\frac{1}{8}\left[\frac{f_{-}^{\prime}(b) b^{2}}{f(b)}-\frac{f_{+}^{\prime}(a) a^{2}}{f(a)}\right] \frac{b-a}{a b}\right) .
$$

These results were obtained in [14].

Let $f:[a, b] \subset(0, \infty) \rightarrow(0, \infty)$ be an $H H$-convex function on the interval $[a, b]$. This is equivalent to the fact that $f$ is $k$-composite- $g^{-1}$ concave on $[a, b]$ 
with $k:(0, \infty) \rightarrow(0, \infty), k(t)=\frac{1}{t}$ and $g(t)=-\frac{1}{t}, t \in[a, b]$. Then by applying Corollary 2 , we have the inequalities

$$
\text { (6.7) } \begin{aligned}
& f\left(\frac{2 a b}{a+b}\right) \\
\leq & \left\{\lambda f^{-1} g^{-1}\left(\frac{2 a b}{(2-\lambda) a+\lambda b}\right)+(1-\lambda) f^{-1}\left(\frac{2 a b}{(1-\lambda) a+(\lambda+1) b}\right)\right\}^{-1} \\
\leq & \left(\frac{a b}{b-a} \int_{a}^{b} \frac{f^{-1}(t)}{t^{2}} d t\right)^{-1} \\
\leq & \left\{\frac{1}{2}\left[f^{-1}\left(\frac{a b}{(1-\lambda) a+\lambda b}\right)+\lambda f^{-1}(a)+(1-\lambda) f^{-1}(b)\right]\right\}^{-1} \leq\left(\frac{f^{-1}(a)+f^{-1}(b)}{2}\right)^{-1}
\end{aligned}
$$

for any $\lambda \in[0,1]$.

By taking the power -1 in (6.7), then we get

$$
\begin{gathered}
\quad f^{-1}\left(\frac{2 a b}{a+b}\right) \\
\geq \lambda f^{-1} g^{-1}\left(\frac{2 a b}{(2-\lambda) a+\lambda b}\right)+(1-\lambda) f^{-1}\left(\frac{2 a b}{(1-\lambda) a+(\lambda+1) b}\right) \\
\geq \frac{a b}{b-a} \int_{a}^{b} \frac{f^{-1}(t)}{t^{2}} d t \\
\geq \frac{1}{2}\left[f^{-1}\left(\frac{a b}{(1-\lambda) a+\lambda b}\right)+\lambda f^{-1}(a)+(1-\lambda) f^{-1}(b)\right] \geq \frac{f^{-1}(a)+f^{-1}(b)}{2}
\end{gathered}
$$

for any $\lambda \in[0,1]$.

If we use Remark 3 , then we get

$$
\begin{aligned}
0 \leq f^{-1}\left(\frac{2 a b}{a+b}\right)-\frac{a b}{b-a} \int_{a}^{b} \frac{f^{-1}(t)}{t^{2}} d t & \\
& \leq \frac{1}{8}\left[\frac{b^{2} f_{-}^{\prime}(b)}{f^{2}(b)}-\frac{a^{2} f_{+}^{\prime}(a)}{f^{2}(a)}\right] \frac{a b}{b-a}
\end{aligned}
$$

and

$$
\begin{aligned}
0 \leq \frac{a b}{b-a} \int_{a}^{b} \frac{f^{-1}(t)}{t^{2}} d t-\frac{f^{-1}(a)+f^{-1}(b)}{2} & \\
& \leq \frac{1}{8}\left[\frac{b^{2} f_{-}^{\prime}(b)}{f^{2}(b)}-\frac{a^{2} f_{+}^{\prime}(a)}{f^{2}(a)}\right] \frac{a b}{b-a}
\end{aligned}
$$

For related results, see [15].

\section{Applications for $p, r$-Convex and LogExp Convex Functions}

If $p>0$ and we consider $g(t)=t^{p}, t \in[a, b] \subset(0, \infty)$, then $f:[a, b] \subset(0, \infty) \rightarrow$ $(0, \infty)$ is $p$-convex on $[a, b]$ is equivalent to the fact that $f$ is composite- $g^{-1}$ convex 
on $[a, b]$. Using Corollary 2 for $k(t)=t$ we get

$(7.1)$

$$
\begin{aligned}
& f\left(M_{p}(a, b)\right) \\
\leq & \lambda f\left[\left(\frac{\lambda b^{p}+(2-\lambda) a^{p}}{2}\right)^{1 / p}\right]+(1-\lambda) f\left[\left(\frac{(1+\lambda) b^{p}+(1-\lambda) a^{p}}{2}\right)^{1 / p}\right] \\
\leq & \frac{p}{b^{p}-a^{p}} \int_{a}^{b} f(t) t^{p-1} d t \\
\leq & \frac{1}{2}\left\{f\left[\left((1-\lambda) a^{p}+\lambda b^{p}\right)^{1 / p}\right]+\lambda f(a)+(1-\lambda) f(b)\right\} \leq \frac{f(a)+f(b)}{2}
\end{aligned}
$$

for any $\lambda \in[0,1]$, where $M_{p}(a, b):=\left(\frac{a^{p}+b^{p}}{2}\right)^{1 / p}$. This improves the corresponding result from [22].

If we use Remark 3 , then we get

$$
0 \leq \frac{p}{b^{p}-a^{p}} \int_{a}^{b} f(t) t^{p-1} d t-f\left(M_{p}(a, b)\right) \leq \frac{1}{8 p}\left(b^{p}-a^{p}\right)\left[\frac{f_{-}^{\prime}(b)}{b^{p-1}}-\frac{f_{+}^{\prime}(a)}{a^{p-1}}\right]
$$

and

$$
0 \leq \frac{a^{p}+b^{p}}{2}-\frac{p}{b^{p}-a^{p}} \int_{a}^{b} f(t) t^{p-1} d t \leq \frac{1}{8 p}\left(b^{p}-a^{p}\right)\left[\frac{f_{-}^{\prime}(b)}{b^{p-1}}-\frac{f_{+}^{\prime}(a)}{a^{p-1}}\right] .
$$

Assume that the function $f:[a, b] \rightarrow(0, \infty)$ is $r$-convex, for $r>0$. This is equivalent to the fact that $f$ is $k$-composite convex with $k(t)=t^{r}, t>0$, and by Corollary 2 for $g(t)=t$ we get

$$
\begin{aligned}
& f\left(\frac{a+b}{2}\right) \\
& \leq\left\{\lambda f^{r}\left(\frac{\lambda a+(2-\lambda) b}{2}\right)+(1-\lambda) f^{r}\left(\frac{(1+\lambda) b+(1-\lambda) a}{2}\right)\right\}^{1 / r} \\
& \leq\left(\frac{1}{b-a} \int_{a}^{b} f^{r}(t) d t\right)^{1 / r} \\
& \leq\left\{\frac{1}{2}\left[f^{r}((1-\lambda) a+\lambda b)+\lambda f^{r}(a)+(1-\lambda) f^{r}(b)\right]\right\}^{1 / r} \leq\left(\frac{f^{r}(a)+f^{r}(b)}{2}\right)^{1 / r}
\end{aligned}
$$

for any $\lambda \in[0,1]$.

By taking the power $r>0$, we get the equivalent inequality

$$
\begin{aligned}
& f^{r}\left(\frac{a+b}{2}\right) \\
& \quad \leq \lambda f^{r}\left(\frac{\lambda a+(2-\lambda) b}{2}\right)+(1-\lambda) f^{r}\left(\frac{(1+\lambda) b+(1-\lambda) a}{2}\right) \\
& \quad \leq \frac{1}{b-a} \int_{a}^{b} f^{r}(t) d t \\
& \quad \leq \frac{1}{2}\left[f^{r}((1-\lambda) a+\lambda b)+\lambda f^{r}(a)+(1-\lambda) f^{r}(b)\right] \leq \frac{f^{r}(a)+f^{r}(b)}{2}
\end{aligned}
$$

for any $\lambda \in[0,1]$. 
From Remark 3, we get for $g(t)=t$ that

$$
\begin{aligned}
0 \leq \frac{1}{b-a} \int_{a}^{b} f^{r}(t) d t-f^{r} & \left(\frac{a+b}{2}\right) \\
& \leq \frac{r}{8}(b-a)\left[f^{r-1}(b) f_{-}^{\prime}(b)-f^{r-1}(a) f_{+}^{\prime}(a)\right]
\end{aligned}
$$

and

$$
\begin{aligned}
0 \leq \frac{f^{r}(a)+f^{r}(b)}{2}-\frac{1}{b-a} & \int_{a}^{b} f^{r}(t) d t \\
& \leq \frac{r}{8}(b-a)\left[f^{r-1}(b) f_{-}^{\prime}(b)-f^{r-1}(a) f_{+}^{\prime}(a)\right] .
\end{aligned}
$$

Assume that $f:[a, b] \rightarrow \mathbb{R}$ is LogExp convex function on $[a, b]$ as considered in [7]. This is equivalent to the fact that $f$ is composite- $g^{-1}$ with $g(t)=\exp t$. By utilising Corollary 2 for $k(t)=t$ we get,

$$
\begin{gathered}
\leq \lambda f\left[\ln \left(\frac{\lambda \exp b+(2-\lambda) \exp a}{2}\right)\right]+(1-\lambda) f\left[\ln \left(\frac{(1+\lambda) \exp b+(1-\lambda) \exp a}{2}\right)\right] \\
\leq \frac{1}{\exp b-\exp a} \int_{a}^{b} f(t) \exp t d t \\
\leq \frac{1}{2}[f[\ln ((1-\lambda) \exp (a)+\lambda \exp (b))]+\lambda f(a)+(1-\lambda) f(b)] \leq \frac{f(a)+f(b)}{2}
\end{gathered}
$$

for $\lambda \in[a, b]$, where $\operatorname{LME}(a, b):=\ln \left(\frac{\exp a+\exp b}{2}\right)$.

If we use Remark 3 , then we get

$$
\begin{aligned}
0 & \leq \frac{1}{\exp b-\exp a} \int_{a}^{b} f(t) \exp t d t-f(\operatorname{LME}(a, b)) \\
& \leq \frac{1}{8}(\exp b-\exp a)\left[\exp (-b) f_{-}^{\prime}(b)-\exp (-a) f_{+}^{\prime}(a)\right]
\end{aligned}
$$

and

$$
\begin{aligned}
0 & \leq \frac{f(a)+f(b)}{2}-\frac{1}{\exp b-\exp a} \int_{a}^{b} f(t) \exp t d t \\
& \leq \frac{1}{8}(\exp b-\exp a)\left[\exp (-b) f_{-}^{\prime}(b)-\exp (-a) f_{+}^{\prime}(a)\right]
\end{aligned}
$$

\section{REFERENCES}

[1] G. D. Anderson, M. K. Vamanamurthy and M. Vuorinen, Generalized convexity and inequalities, J. Math. Anal. Appl. 335 (2007) 1294-1308.

[2] N. S. Barnett, P. Cerone and S. S. Dragomir, Some new inequalities for HermiteHadamard divergence in information theory, in Stochastic Analysis \& Applications, Vol. 3, Eds. Y. J. Cho, J. K. Kim and Y. K. Choi, pp. 7-19, Nova Sci. Publishers, 2003, ISBN 1-59033-860-X. Preprint RGMIA Res. Rep. Coll. 5 (2002), Issue 4, Art. 8. [Online http://rgmia.org/papers/v5n4/NIHHDIT .pdf] .

[3] E. F. Beckenbach, Convex functions, Bull. Amer. Math. Soc. 54 (1948), 439-460.

[4] S. S. Dragomir, An inequality improving the first Hermite-Hadamard inequality for convex functions defined on linear spaces and applications for semi-inner products, J. Inequal. Pure Appl. Math. 3 (2002), No. 2, Article 31. [Online https://www.emis.de/journals/JIPAM/article183.html?sid=183] . 
[5] S. S. Dragomir, An inequality improving the second Hermite-Hadamard inequality for convex functions defined on linear spaces and applications for semi-inner products, J. Inequal. Pure Appl. Math. 3 (2002), No. 3, Article 35. [Online https://www . emis.de/journals/JIPAM/article187.html?sid=187] .

[6] S. S. Dragomir, Ostrowski type inequalities for Lebesgue integral: a survey of recent results. Aust. J. Math. Anal. Appl. 14 (2017), no. 1, Art. 1, 283 pp. [Online http://ajmaa.org/cgi-bin/paper.pl?string=v14n1/V14I1P1.tex] .

[7] S. S. Dragomir, Inequalities for a generalized finite Hilbert transform of convex functions, Preprint RGMIA Res. Rep. Coll. 21 (2018), Art.

[8] S. S. Dragomir and C. E. M. Pearce, Selected Topics on HermiteHadamard Inequalities and Applications, RGMIA Monographs, 2000. [Online http://rgmia.org/monographs/hermite_hadamard.html] .

[9] S. S. Dragomir, New inequalities of Hermite-Hadamard type for log-convex functions. Khayyam J. Math. 3 (2017), no. 2, 98-115.

[10] S. S. Dragomir, Inequalities of Hermite-Hadamard type for $G A$-convex functions, to appear in Annales Mathematicae Silesianae, Preprint RGMIA Res. Rep. Coll. 18 (2015), Art. 30. [Online http://rgmia.org/papers/v18/v18a30.pdf] .

[11] S. S. Dragomir, Inequalities of Hermite-Hadamard type for $G G$-convex functions, Preprint RGMIA, Research Report Collection, 18 (2015), Art. 71, 15 pp., [Online http://rgmia.org/papers/v18/v18a71.pdf] .

[12] S. S. Dragomir, Some integral inequalities of Hermite-Hadamard type for $G G$-convex functions, Mathematica (Cluj), 59 (82), No 1-2, 2017, pp. 47-64. Preprint RGMIA, Research Report Collection, 18 (2015), Art. 74. [Online http://rgmia.org/papers/v18/v18a74.pdf] .

[13] S. S. Dragomir, Inequalities of Hermite-Hadamard type for $H A$-convex functions, Maroccan J. Pure 83 Appl. Analysis, Volume 3 (1), 2017, Pages 83-101. Preprint, RGMIA Res. Rep. Coll. 18 (2015), Art. 38. [Online http://rgmia.org/papers/v18/v18a38.pdf] .

[14] S. S. Dragomir, Inequalities of Hermite-Hadamard type for $H G$-convex functions, Probl. Anal. Issues Anal. Vol. 6 (24), No. 2, 2017 1-17. Preprint, RGMIA Res. Rep. Coll. 18 (2015), Art. 79. [Online http://rgmia.org/papers/v18/v18a79.pdf].

[15] S. S. Dragomir, Inequalities of Hermite-Hadamard type for $H H$-convex functions, to appear in Acta et Commentationes Universitatis Tartuensis de Mathematica, Preprint, RGMIA Res. Rep. Coll. 18 (2015), Art. 80. [Online http://rgmia.org/papers/v18/v18a80.pdf] .

[16] A. El Farissi, Simple proof and refinement of Hermite-Hadamard inequality, J. Math. Ineq. 4 (2010), No. 3, 365-369.

[17] L. Fejér, Über die Fourierreihen, II, (In Hungarian) Math. Naturwiss, Anz. Ungar. Akad. Wiss., 24 (1906), 369-390.

[18] D. S. Mitrinović and I. B. Lacković, Hermite and convexity, Aequationes Math. 28 (1985), 229-232.

[19] P. Montel, Sur les functions convexes et les fonctions sousharmoniques, Journal de Math., 9 (1928), 7, 29-60.

[20] C. P. Niculescu, Convexity according to the geometric mean, Math. Inequal. Appl., 3, (2000), 2, $155-167$.

[21] C. E. M. Pearce, J. Pečarić and V. Šimić, Stolarsky means and Hadamard's inequality. J. Math. Anal. Appl. 220, 99-109 (1998)

[22] K. S. Zhang and J. P. Wan, p-convex functions and their properties. Pure Appl. Math. 23(1), 130-133 (2007).

${ }^{1}$ Mathematics, College of Engineering \& Science, Victoria University, PO Box 14428 , Melbourne City, MC 8001, Australia.

E-mail address: sever.dragomir@vu.edu.au

$U R L:$ http://rgmia.org/dragomir

${ }^{2}$ DSt-nRF Centre of Excellence in the Mathematical, and Statistical Sciences, School of Computer Science, \& Applied Mathematics, University of the WitwaterSrand, Private Bag 3, Johannesburg 2050, South Africa 